\title{
PORTUGUÊS BRASILEIRO E ALEMÃO EM CONTATO EM DUAS COMUNIDADES VIRTUAIS
}

\author{
Layla Cristina Iapechino Souto
}

(Doutoranda da Europa-Universität Viadrina)

RESUMO

Este artigo apresenta uma análise de ocorrências de code-switching entre as línguas Português Brasileiro e Alemão em duas comunidades virtuais no Facebook: Brasileiros em Berlim e Brasileiros e Brasileiras em Berlim. Adotamos os conceitos de durabilidade, permeabilidade e liminalidade traçados por Zinkhahn-Rhobodes (2015) para observarmos a permeabilidade da fronteira linguística entre essas duas línguas.

PALAVRAS-CHAVE: línguas em contato; code-switching; fronteira linguística.

\section{Introdução}

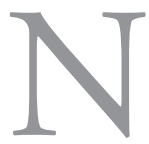

os séculos XIX e XX a história relata uma enorme imigração de famílias alemãs para o Brasil à procura de melhores condiçôes de vida. No século XXI este quadro se inverteu, e é a Alemanha que agora recebe um número crescente de imigrantes brasileiros. A Alemanha é um país plurilíngue e pluricultural. De acordo com dados do Destatis, publicados em setembro de 2014 , cerca de 7,401 milhóes ${ }^{1}$ de estrangeiros vivem no país. Se se considerar o número de descendentes de estrangeiros que foram para a Alemanha após 1950, ultrapassa-se um total de 16 milhões. Só em Berlim, de acordo com o Amt für Statistik Berlin-Brandenburg (junho de 2012)2 ${ }^{2}$, cidadãos de mais de

1 https://www.destatis.de/DE/ZahlenFakten/GesellschaftStaat/Bevoelkerung/Bevoelkerungsstand/Tabellen/Zensus_Geschlecht_Staatsangehoerigkeit.html

2 https://www.berlin.de/lb/intmig/statistik/demografie/einwohner_staatsangehoerigkeit. html 
80 nacionalidades convivem na cidade, fazendo com que sua sociedade seja multiétnica, multicultural e multilíngue.

Com base nos Relatórios Consulares enviados anualmente pelos Consulados e Embaixadas sobre o número de brasileiros residentes em suas respectivas jurisdiçóes, o Itamaraty aponta que, em 2013, havia $113.310 \mathrm{mil}$ brasileiros oficialmente registrados na Alemanha ${ }^{3}$. A estimativa do Itamaraty destaca que esse número pode ser duplicado se considerarmos os brasileiros não oficialmente registrados e os que têm dupla cidadania e que vivem como europeus.

Ao contrário do volume de pesquisas que se debruçam sobre o contato entre o português brasileiro (PB) e o alemão (AL) e seus dialetos no Brasil (fruto da imigração alemã que se iniciou no início do século XIX), há poucos estudos sobre o contato entre essas duas línguas em terras germânicas. Diante desses números, e diante dos poucos estudos sobre o contato entre o $\mathrm{PB}$ e o AL na Alemanha, despertou-nos o interesse de acompanhar mais de perto a comunidade migrante brasileira na cidade de Berlim, a fim de observar o contato linguístico entre essas duas línguas.

Para essa exposição, analisamos as manifestaçóes de code-switching em duas comunidades brasileiras no facebook: Brasileiros em Berlim e Brasileiros e Brasileiras em Berlim. Mais especificamente, utilizamos os conceitos de durabilidade, permeabilidade e liminalidade apresentados por Zinkhahn-Rhobodes (2015), a fim de observar a permeabilidade da "fronteira linguística” 45 entre o PB e o AL manifestada em conversas nessas duas comunidades virtuais.

Iniciamos o artigo apresentando uma revisão dos pressupostos teóricos que norteiam essa pesquisa, a saber, linguas em contato e code-switching, durabilidade, permeabilidade e liminalidade. Em seguida, apresentamos a metodologia utilizada bem como a análise dos dados coletados no Facebook. Por fim, apresentamos uma breve discussão.

3 http://www.brasileirosnomundo.itamaraty.gov.br/a-comunidade/estimativas-populacionais-das-comunidades/estimativas-populacionais-das-comunidades-brasileiras-no-mundo-2013/Estimativas\%20Brasileiros\%20no\%20Mundo\%202013.pdf

4 "language borders" (Zinkhahn-Rhobodes, 2015, p. 229).

5 Todas as traduçóes apresentadas neste artigo são de responsabilidade da autora. 


\section{Linguas em contato e o code-switching}

O estudo sobre línguas em contato teve sua gênese com a publicação da obra Languages in contact em 1953, por Weinreich, onde o autor defendeu que qualquer estudo sobre línguas em contato deve considerar não apenas os fatores internos da língua, mas também a comunidade em que as duas ou mais línguas são faladas. Com base em outros estudos, ele demonstrou que as consequências do contato entre línguas são, em boa parte, condicionadas por variáveis socioculturais, que incluem o grau de bilinguismo dos falantes, o tempo de contato entre as línguas, a distribuição geográfica e demográfica, os fatores sociais (como religião, raça, gênero e idade), o uso da língua em diferentes contextos sociais (como na educação, no governo, na mídia e na literatura) e fatores políticos e ideológicos (que incluem prestígio e "language loyalty") (WEINREICH, 1953, p. 106).

Apesar de haver inúmeras investigaçôes sobre fenômenos de línguas em contato antes da década de 1950, foi apenas nessa época que houve uma sistematização teórica. Em pesquisas mais antigas, as conclusóes baseavam-se na intuição dos linguistas, o que não era suficiente para construir uma teoria que abarcasse padrōes científicos ${ }^{6}$.

Em linhas gerais, definem-se línguas em contato como uma situação em que línguas ou variedades de línguas entram em contato umas com as outras devido à proximidade geográfica ou social, migraçóes, conquistas, exposição aos meios de comunicação em massa e quando falantes de diferentes línguas ou variedades se misturam em uma mesma comunidade (MARANHÃO, 2011; THOMASON, 2001). O fenômeno do contato linguístico não está restrito a algumas línguas, sendo norma para toda e qualquer língua ${ }^{7}$.

6 Apesar da recente sistematização teórica, o contato linguístico não é restrito às sociedades atuais. Segundo Thomason (2001, p. 8), "more than enough information is available to show that extensive and intricate language contacts, with far-reaching social, political, and linguistic effects, are a constant feature of the human condition, not a phenomenon that is limited to large, recent, militarily prominent, andlor technologically advanced societies". Em seu livro, a autora cita exemplos de contato linguístico antes de Cristo. (Cf. THOMASON, 2001, p. 3-8)

7 "language contact is the norm, not the exception. We would have a right to be astonished if we found any language whose speakers had successfully avoided contacts with all other languages for periods longer than one or two hundred years" (Thomason, 2001, p. 10). 
Muhvic-Dimanovski (2009) defende que muitos fatores são extremamente importantes no processo de integração de elementos estrangeiros em uma língua, e esses fatores devem ser estudados aprofundadamente. Para a autora, "questôes como quem está emprestando uma palavra, por que, e como, precisam ser respondidas antes que se possa ter uma visão completa do problema" (MUHVIC-DIMANOVSKI, 2009, p. 53). O resultado, então, do contato de uma língua $A$ com uma língua $B$ não será uma língua $A B$, mesmo que ambas as línguas adotem elementos uma da outra, o resultado será sempre $\mathrm{A}^{\mathrm{B}}$ ou $\mathrm{B}^{\mathrm{A}}$ (MUHVIC-DIMANOVSKI, 2009, p. 53).

Os estudos que tratam de línguas em contato se baseiam na noção de bilinguismo e consideram o falante bilíngue como o objeto de pesquisa sobre a interação entre diferentes padróes linguísticos. Antigamente só era considerado bilíngue o falante que apresentasse domínio total em ambas as línguas, o que é relativamente raro. Esse conceito foi substituído pela ideia de que bilíngue é o falante que pode produzir pelo menos algumas sentenças em outra língua. Assim, bilinguismo passou a ser visto como um fenômeno relativo que afeta a maioria da população mundial (SAVEDRA, 2009).

Conectados ao bilinguismo, desenvolveram-se estudos sobre o code-switching, que, em linhas gerais, é o uso alternado de dois ou mais sistemas linguísticos por um falante bilíngue em uma mesma conversação, e frequente em diálogos entre bilíngues (PORTO, 2007; POPLACK, 1980). Falantes bilíngues (ou multilíngues) usam, entretanto, suas diferentes línguas de maneiras diferentes para transmitir suas ideias em uma interação com outros bilíngues por várias razóes. As estratégias por eles utilizadas no emprego de uma ou outra(s) língua(s) têm sido um campo fértil de investigação dentro da linguística, psicolinguística, sociolinguística e neurolinguística.

Em sua gênese, os primeiros estudos sobre o code-switching tinham como objetivo elaborar tipologias funcionais sobre esse fenômeno, numa abordagem "tradicional"' (PORTO, 2007). Gumperz (1992), por exemplo, a partir de dados de três comunidades, com os pares linguísticos espanhol-inglês, esloveno-alemão

8 "questions as who is borrowing a word, where, why, and how have to be answered before one can have a complete view of the problem".

9 Muito da literatura sobre o code-switching dedica-se às funçóes sócio-pragmáticas do code-switching. Autores geralmente citados dentro dessa tradição são: Gumperz, 1976; Gumperz \& Hernándes-Chável, 1970; Grosjean, 1982; Appel \& Muysken, 1987; entre outros. 
e hindu-inglês, listou seis funçóes de code-switching: quotation, addressee specification, interjection, repetition, message qualification, personification or objectification.

Conforme os estudos foram se desdobrando, a essa lista foram agrupadas outras funções, como é observado nos trabalhos de Grosjean (1982), Appel e Muysken (1987), Koziol (2000) e Richardson (2000). De um modo geral, essas listas são descriçốes taxonômicas, distantes ainda de explicar o comportamento do code-switching.

Em nível funcional, Barredo (2001, p. 528) destaca que o code-switching, "é geralmente usado como uma estratégia comunicativa e, portanto, pode expressar várias e diferentes funçóes dentro do discurso”, e, em nível estrutural, "vários estudos fornecem evidências de que certas restrições linguisticas governam o code-switching". ${ }^{10}$

As listas de funçóes do code-switching foram questionadas por pesquisadores, como Auer (1984, p. 3), que, logo nos primeiros anos desses estudos, diz:

"Meramente enumerar tipos de alternância da linguagem parece inadequado por um número de razóes. Para começar, é um esforço inútil dar um esquema fechado de classificação para o code-switching, para um número indeterminado de interpretaçôes que se pode chegar. $\mathrm{O}$ que exatamente um participante bilíngue faz quando ele ou ela alterna as línguas está intimamente ligado às circunstâncias específicas e não-idênticas na qual a alternância ocorre." ${ }^{11}$

Por outro lado, encontramos estudos, como Poplack (1980, 1981), que defendem que, para além das motivações sócio-pragmáticas, fatores linguísticos também determinam o uso do code-switching, assim como Auer (1998, p. 2), para quem

10 "is often used as a communicative strategy and therefore, can express several and different functions within the discourse" [...] "several studies provide evidence that certain linguistic constraints govern code-switching”.

11 "Merely enumerating types of language alternation seems inadequate for a number of reasons. To begin with, it is a futile endeavor to give a closed classificational scheme for codeswitching, for a indeterminate number of interpretations can be arrived at. What exactly a bilingual participant is doing when he or she switches languages is closely tied to the specific, never-identical circumstances in which alternation occurs". 
"Embora as línguas envolvidas no code-switching possam indexar algum tipo de conhecimento extra-conversacional, code-switching não pode ser analisado como uma simples consequência de tais indexações, sem levar em conta a posição sequencial em que ocorre e da qual recebe o seu significado." ${ }^{2}$

Meyers-Scotton (1993), por sua vez, considerou não só as funções de mudança de código dentro do discurso, mas também o papel que o code-switching tem dentro dos padróes de escolha linguística em uma comunidade. Assim, sob esse olhar, o code-switching é relacionado às interaçóes pragmáticas do falante e é considerado como uma tática comunicativa relevante no discurso. De acordo com Porto (2007: 2), este fenômeno não é aleatório e tampouco é consequência de falta de proficiência nas línguas envolvidas, "uma vez que o enunciado híbrido resultante do code-switching possui motivaçóes e funções sócio-pragmáticas e está sujeto a restrições gramaticais”.

\section{Os conceitos de durabilidade, permeabilidade e liminalidade}

Para sua pesquisa de doutorado, Zinkhahn-Rhobodes analisou o contato linguístico entre o polonês e o alemão nas cidades de Frankfurt (Oder) e Stubice, localizadas na fronteira entre a Alemanha e a Polônia. A autora observou que o contato entre o alemáo e o polonês gerou muitas formas linguísticas mixas, observadas no dia a dia da comunidade, mostrando, segundo ela, "que o uso da linguagem em contextos multilíngues fornece um âmbito para permeabilidade entre os sistemas linguísticos"13 (ZINKHAHN-RHOBODES, 2015, p. 230).

O intenso contato entre duas línguas, como o observado pela pesquisadora (2015, p. 230), "leva à convergência - e talvez fusão - de estruturas linguísticas morfossintáticas, e, em consequência, à emergência de formas lin-

12 "although the languages involved in code-switching may index some kind of extra-conversational knowledge, code-switching can never be analysed as a mere consequence of such indexing, without taking into account the sequential position in which it occurs and from which it receives its meaning".

13 "that language use in multilingual contexts provides a scope for permeability between language systems" 
guísticas híbridas, que pode até levar à dissolução de fronteiras linguísticas”. ${ }^{14}$ Fronteira linguística ${ }^{15}$ é definida pela pesquisadora como "a borda estrutural entre dois sistemas linguísticos, na maioria das vezes manifestado foneticamente como o local de alternância linguística” ${ }^{16}$

Com base em pesquisas ${ }^{17}$ que investigaram o code-switching como uma ultrapassagem da fronteira linguística, Zinkhahn-Rhobodes (2015) traçou os conceitos de Durabilidade, Permeabilidade e Liminalidade ${ }^{18}$. Segundo a pesquisadora, durabilidade se refere a barreiras estáveis e concretas entre as línguas. Nesse caso, as barreiras são impermeáveis e não há, portanto, influência nos níveis fonéticos, morfológicos e sintáticos entre as línguas. A fronteira entre uma língua e outra é claramente observável (ZINKHAHN-RHOBODES, 2015, p. 233-234).

Por sua vez, permeabilidade se refere à porosidade observável na fronteira entre uma língua e outra. Nesse caso, apesar de uma fronteira observável entre as línguas, há permeabilidade, o que permite a ultrapassagem de interferência nos níveis fonéticos, morfológicos e/ou sintáticos (ZINKHAHN-RHOBODES, 2015, p. 234)

Por fim, liminalidade refere-se a uma área de transição, uma zona fronteiriça ${ }^{19}$, entre uma língua e outra. Nesse "espaço liminal”" não é possível observar claramente a fronteira entre as línguas, assim, "a classificação e a atribuição de elementos linguísticos a uma língua ou a outra se torna difícil" 21 (ZINKHAHN-RHOBODES, 2015, p. 236).

14 "leads to convergence - and maybe fusion - of morphosyntactic language structures, and in consequence to the emergence of hybrid language forms, it may even lead to the dissolution of language borders".

15 O termo usado por Zinkhahn-Rhobodes (2015) é "language border", que aqui traduzimos por "fronteira linguística".

16 "the structural border between two languages systems, mostly phonetically manifested as the site of language switch".

17 Ver Hinnenkamp \& Meng (2005), Cunha et al. (2012), Rampton (2005).

18 Durability, Permeability e Liminality (Zinkhahn-Rhobodes, 2015)

19 "Border zones" (Zinkhahn-Rhobodes, 2015: 235).

20 Zinkhahn-Rhobodes (2015) opta pelo termo "liminal space", que traduzimos aqui como "espaço liminal".

21 "The classification and allocation of linguistic elements to one language or the another becomes difficult". 
$\mathrm{Na}$ figura abaixo, reproduzimos os esquemas apresentados pela pesquisadora para ilustrar os conceitos de durabilidade, permeabilidade e liminalidade.

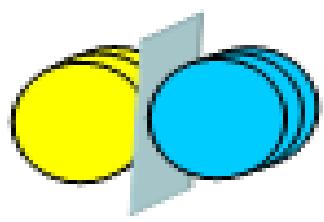

Durabilidade

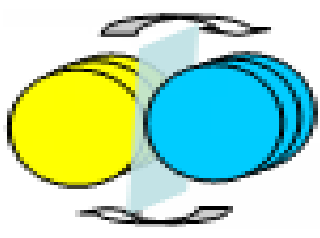

Permeabilidade

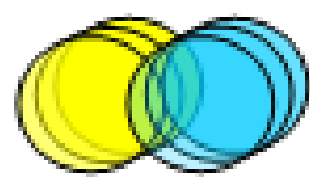

Liminalidade

Figura 1: Durabilidade, permeabilidade e liminalidade na fronteira linguística, segundo Zinkhahn-Rhobodes (2015, p. 234-235)

De acordo com Zinkhahn-Rhobodes (2015, p. 233), os três círculos em cada ilustração se referem aos três níveis de análise por ela adotados, a saber: fonético, morfológico e sintático. Nas seções que seguem, apresentamos a metodologia adotada para a coleta de dados e a análise do code-switching com base nos conceitos de durabilidade, permeabilidade e liminalidade.

\section{Metodologia}

Para essa pesquisa, concentramos nossa coleta de dados na rede social Facebook. Mais especificamente, selecionamos conversas entre brasileiros em duas comunidades: Brasileiros em Berlim e Brasileiros e Brasileiras em Berlim. A primeira comunidade conta com 8.317 membros $^{22}$ e tem como objetivo “'agrupar Brasileiros de todas as naçóes' que estão, estiveram ou estarão em Berlin. [...] A comunidade visa, mas não se limita, à troca de experiências e integração além de oferecer um espaço para pessoas se conhecerem e trocarem experiências".

Também a comunidade Brasileiros e Brasileiras em Berlim, que tem 5.473 membros ${ }^{23}$, tem em sua descrição: "Este é um grupo voltado a brasileiros e brasileiras que já residem, já residiram ou desejam residir em Berlim ou simpatizantes desta cidade, visando a troca de informaçóes e experiências e a busca de novas contatos e amizades".

$\begin{array}{ll}{ }^{22} & \text { No dia } 18 / 03 / 2016 \\ 23 & \text { No dia } 18 / 03 / 2016 .\end{array}$ 
De ambas as comunidades, selecionamos publicaçóes que revelam code-switching para compor nosso corpus de análise. Apesar de esses dados estarem disponíveis em um meio de comunicação escrito, a concepção linguística é muito próxima da linguagem falada, fazendo desse gênero textual um gênero híbrido (MARCUSCHI, 2002). Portanto, pode-se supor que fenômenos da fala são reproduzidos nessa interação virtual e os dados obtidos são válidos para uma análise.

\section{Análise dos dados}

Propomos, neste artigo, uma análise de ocorrências de code-switching entre PB e AL observadas em publicaçóes de brasileiros e brasileiras em duas comunidades brasileiras no Facebook, e, para isso, utilizamos os conceitos de durabilidade, permeabilidade e liminalidade traçados por Zinkhahn-Rhobodes (2015). Não encontramos, entre os exemplos, nenhum caso de liminalidade e, portanto, apresentamos aqui casos de durabilidade e permeabilidade, que se dão em diferentes pontos da estrutura gramatical.

\section{Durabilidade}

Nos casos de durabilidade observados em nosso corpus, o discurso alterna entre o $\mathrm{PB}$ e o $\mathrm{AL}$, mas a alteração é claramente de uma língua para a outra e o limite entre uma língua e outra é evidentemente mantido, como podemos verificar no exemplo (01):

(1) Ok não pode unterschätzen os terroristas... Mas gente! Eles querem exatamente isso o caos, o pânico, a destruição [...] Como já disse, eles sao um perigo? Sim! Pode unterschätzen? Não!

(Ok não pode subestimar os terroristas... Mas gente! Eles querem exatamente isso o caos, o pânico, a destruição [...] Como já disse, eles sao um perigo? Sim! Pode subestimar? Não!)

Em (01), a fronteira entre o PB e o AL é claramente mantida. Apesar de a informante emprestar a palavra unterschätzen do léxico alemão, não há influência morfológica e/ou sintática, e, assim, a impermeabilidade da fronteira linguística é mantida. 
Ocorrências como (01) não obedecem à Restrição de Equivalência de Poplack (1980, p. 586), que diz: "code-switches tendem a ocorrer em pontos no discurso onde a justaposição de elementos de L1 e L2 não viola uma regra sintática de uma ou outra língua". ${ }^{24} \mathrm{Em}(01)$, a frase "não pode unterschätzen os terroristas" possui o verbo auxiliar "pode", o que levaria o verbo principal "unterschätzen” a ocupar o último lugar na oração, já que, em frases afirmativas na sintaxe alemã, quando se usa um verbo auxiliar, o verbo principal passa a ocupar, em sua forma infinitiva, a última posição da frase, como podemos ver em (02).

(2) Man kann nicht die Terroristen unterschätzen.

(Se pode não os terroristas subestimar $^{25}$

\section{Permeabilidade}

$\mathrm{Na}$ permeabilidade, a fronteira entre as línguas é porosa. Apesar de ainda serem observáveis, como na durabilidade, elas são passíveis de serem trespassadas. De acordo com Zinkhahn-Rhobodes (2015, p. 234), "elas constituem um limite penetrável que permite um impacto fonético, morfológico e/ou sintático reciproco entre duas línguas em contato" ${ }^{26}$

O exemplo (02) ilustra a fronteira permeável entre o PB e o AL. O code-switching, nesse caso, ocorre entre o limite lexical de uma língua e outra:

(3) Consigo fazer uma outra Ausweis [identidade] alemã e caso sim, preciso pagar alguma taxa?

Quando eu for no consulado, além de informar o ocorrido, preciso eu pedir uma nova identidade brasileira?

No PB, substantivos podem ser formados através de outras classes de palavras e são femininos quando possuem o sufixo -dade. No exemplo (02), a palavra identidade (de origem latina), é um substantivo formado a partir do advérbio idem, que significa $o$ mesmo, e o sufixo -dade, que indica estado ou

24 "code-switches will tend to occur at points in discourse where juxtaposition of L1 and L2 elements does not violate a syntactic rule of either language".

25 Tradução palavra por palavra.

26 "They constitute a penetrable threshold which enables reciprocal phonetic, morphological and/or syntactic impact between two languages in contact". 
qualidade, recebendo, assim, o traço [+fem]. Em alemão, a palavra Ausweis é masculina, der Ausweis (a identidade).

De acordo com Freitas et al (2003, p. 6), “o traço [- fem] é atribuído por defeito, quando não existe qualquer tipo de motivação formal ou semântica para atribuir o traço [+ fem] à palavra”. Entretanto, quando há atração sinonímica, processo pelo qual a palavra estrangeira é associada a uma palavra vernácula que designa um conceito equivalente, a palavra estrangeira adquire o gênero de sua correspondente. Sendo assim, o traço [+fem] é atribuído à palavra alemã "Ausweis" pela atração sinonímica com sua correspondente em $\mathrm{PB}$, “a identidade”, como é ilustrado na figura 2:

\begin{tabular}{cc}
\hline A identidade & Der Ausweis \\
\hline $\begin{array}{c}\text { "Idem"+dade } \\
{[+ \text { fem }]}\end{array}$ & {$[-\mathrm{fem}]$} \\
... uma outra Ausweis alemã.
\end{tabular}

Figura 2: Permeabilidade: a identidade/der Ausweis

Como mostra a figura 2, podemos observar uma fronteira permeável entre o PB e o AL. Apesar de usar a palavra em AL, Ausweis, o gênero permanece como na correspondente em $\mathrm{PB}$, a identidade

O mesmo acontece no exemplo (04):

(4) Boa noite Pessoal, tudo bem? Em breve farei meu anmeldung e queria saber onde é o melhor lugar para fazer o meu registro, alguma indicação?

Apesar de "Anmeldung" ser uma palavra feminina ${ }^{27}$, ela adquire o traço [-fem] de sua correspondente em $\mathrm{PB}$, o registro, como ilustrado na figura 3:

\begin{tabular}{|c|c|}
\hline Die Anmeldung & $\mathrm{O}$ registro \\
\hline$[+\mathrm{fem}]$ & [-fem] \\
\hline \multicolumn{2}{|c|}{...meu anmeldung } \\
\hline
\end{tabular}

Figura 3: Permeabilidade: die Anmeldung / o registro.

27 "Feminina sind Wortformen, die auf Suffixe oder typische Wortausgänge enden: [...] Ung: die Achtung, die Bildung, die Zeitung, Ausnahme: der Hornung" (Darski, 2010, p. 270). 
$\mathrm{Na}$ mesma publicação onde retiramos o exemplo (03), a informante publicou a seguinte frase:

(5) $I_{a}$ Quando eu for no consulado, além de informar o ocorrido, $I_{b}$ preciso eu pedir uma nova identidade brasileira ${ }_{b}$ ?

Aqui temos outro exemplo de fronteira permeável. Em (05) há uma oração temporal (com valor temporal e condicional) ${ }^{28}$ [a] que precede a oração principal [b]. Segundo Brito (2006, p. 724), as oraçóes temporais com quando se comportam como oraçóes subordinadas adverbiais, que, por sua vez, podem ocupar diferentes posições na frase. ${ }^{29}$ Quando a oração temporal ocupa a posição inicial, a oração principal, devido ao caráter Sujeito-Verbo-Objeto do $\mathrm{PB}$, deve ser iniciada pelo sujeito ou pelo sujeito nulo da frase. Já em alemão, uma oraçáo condicional segue a estrutura: $\left[_{\text {oraçáo condicional }}\right.$ wenn + Sujeito + Objeto + Verbo], [oraçăo principal Verbo + Sujeito + Objeto $]$.

No exemplo (05) podemos ver claramente uma interferência do AL na ordem de palavras da oração principal, preciso eu..., onde a informante inicia a oração principal com o Verbo, seguindo a estrutura da gramática alemã para a oração principal que ocorre após a oração condicional: ... , [ ${ }_{\text {oraçáo principal }}$ Verbo + Sujeito + Objeto].

Por fim, o exemplo (06) ilustra uma ocorrência de code-switching dentro do limite do morfema:

\section{(6) Foi assim que fiz quando me anmeldei aqui.}

No AL, há apenas uma conjugação verbal, “-en”, e no $\mathrm{PB}, 3$ conjugaçôes, “-ar, -er, -ir”. Em PT, a primeira delas é regular e, assim, os neologismos verbais tendem a adotar a primeira conjugação. No exemplo (06), a palavra

28 De acordo com Brito (2006, p. 723), “Quando, além do valor temporal, pode ter outros valores: (a) temporal e condicional (...) (b) temporal e concessivo(...)”.

29 "Posição inicial, posição final, posição medial:

(a) Se a economia e o mercado forem regulados poderão contribuir para um mundo mais justo.

(b) A economia e o mercado contribuirão para um mundo mais justo se forem regulados.

(c) A economia e o mercado, se forem regulados, contribuirão para um mundo mais justo."

(BRITO, 2006, p. 698). 
anmeldei é criada a partir da raiz do verbo em alemão, anmelden ${ }^{3 \theta}$, e a terminação para primeira pessoa do singular no pretérito perfeito "ei”, como ilustra a figura 4:

\begin{tabular}{ccc}
\hline en & ar \\
\hline $\begin{array}{c}\text { anmelden } \\
\text { anmeld }\end{array}$ & $1^{\text {a }}$ p.s. Pret. Perf. \\
& ei \\
& anmeldei & \\
\hline
\end{tabular}

Figura 4: Code-switching dentro do limite do morfema.

Em casos como (06), apenas falantes de $\mathrm{PB}$, que estão inseridos num contexto onde o AL é falado, apropriam-se do significado desse neologismo. Apesar de esse exemplo apresentar aspectos de liminalidade, podemos notar que a fronteira linguística é ainda mantida, já que a fronteira entre o léxico e o morfema de ambas as línguas é facilmente identificável.

\section{Conclusões}

Neste artigo, analisamos algumas manifestações de code-switching em duas comunidades brasileiras no facebook: Brasileiros em Berlim e Brasileiros e Brasileiras em Berlim. Para essa análise, utilizamos os conceitos de durabilidade, permeabilidade e liminalidade apresentados por Zinkhahn-Rhobodes (2015) a fim de observar a permeabilidade da fronteira linguística entre o PB e o AL.

Nas ocorrências de code-switching retiradas dos grupos, observamos casos que apresentam fronteiras duráveis e permeáveis nos níveis morfológicos e sintáticos entre o PB e o AL. Nos casos de fronteira durável, as barreiras entre os dois sistemas linguísticos se mostram estáveis, sem influência entre uma língua e outra, enquanto que nos casos de fronteira permeável podemos observar interferências nos níveis sintáticos e morfológicos entre o PB e o AL.

30 "Wir erhalten den indikativischen präsentischen Flexionsstamm durch das Wegstreichen der Buchstaben $\boldsymbol{e n}$ oder $\boldsymbol{n}$ von einfachen Infinitiv. Nur der Buchstabe $\boldsymbol{n}$ wird gestrichen, wenn der einfache Infinitiv nicht auf en (Ausnahme: knien) ausgeht” (Darski, 2010, p. 201). 
Não encontramos, nos diálogos online observados em ambas as comunidades, nenhum caso definido de liminalidade. Não excluímos, entretanto, a possível existência de casos de espaços liminais passíveis de serem observados em conversas entre brasileiros bilíngues (PB e AL). Nesse sentido, pretendemos estender essa pesquisa para a observação do contato linguístico entre o PB e o AL na fala de brasileiros bilíngues residentes na Alemanha, o que possibilitará também a observação da fronteira linguística no nível fonológico.

\section{Referências}

APPEL, Rene; MUYSKEN, Pieter. Language contact and bilingualism. London: Edward Arnold, 1987.

AUER, Peter. Bilingual conversation. Amsterdam/Philadelphia: John Benjamins, 1984.

. From Code-switching via Language Mixing to Fused Lects: Toward a Dynamic Typology of Bilingual Speech. In: InLISt Interaction and Linguistic Structures, n. 6, set/1998.

BARREDO, Inma Muñoa. Pragmatic functions of code-switching among basque-spanish bilinguals. Atas do I Simpósio Internacional sobre o Bilinguismo, 2001.

BRITO, Ana Maria. Subordinação adverbial. In: Mateus et al. (eds.) Gramática da Lingua Portuguesa (7 $\left.7^{\mathrm{a}} \mathrm{ed}\right)$. Lisboa: Caminho, 2006.

CUNHA, Conceição et al. (eds.) Über Granyen sprechen. Mehsprachgkeit in Europa und der Welt. Würzburg: Königshausen \& Neumann, 2012.

DARSKI, Józef Paweł. Deutsche Grammatik. Frankfurt am Main: Peter Lang, 2010.

FREITAS, Tiago/RAMILO, Maria Celeste/SOALHEIRO, Elisabete. O processo de integração dos estrangeirismos no português europeu, CELGA, 2003. GROSJEAN, François. Life with two languages: an introduction to bilingualism. Cambridge, MA: Harvard University Press, 1982.

GUMPERZ, John. Discourse strategies. Cambridge, UK: Cambridge University Press, 1982.

HINNENKAMP, Volker; MENG, Katharina (eds.). Sprachgrenzen überspringen. Sprachliche Hybridität und polykulturelles Selbstverständnis. Tübingen: Narr, 2005. 
JAKOBSON, Roman. Closing Statement: Linguistics and Poetics. In: T. A. Sebeok (Ed.) Style in language. Cambridge, MA: MIT Press, 1960, p. 350-377.

JUNGBLUTH, Konstanze. Aus zwei mach eins: switching, mixing, getting different. In: JANCZAK, Barbara; JUNGBLUTH, Konstanze; WEYDT, Harald. (eds.). Mehsprachgkeit aus deutscher Perstpektive. Tübingen, 2012.

KOZIOL, Jessica Marie. Code-switching between Spanish and English in contemporary American society. Monografia (Inglês e Língua Estrangeira). Maryland: St. Mary's College of Maryland, 2005.

MARCUSCHI, Luiz Antônio. Gêneros textuais: definição e funcionalidade. In: DIONISIO, A. P.; MACHADO, A. R.; BEZERRA, M. A. (Orgs.) Gêneros textuais e ensino. Rio de Janeiro: Lucerna, 2002.

MUHVIC-DIMANOVSKI, Vesna. Languages in contact. Linguistic Anthropology, vol. 1, 2009, 245p. pp. 52-62.

MYERS-SCOTTON, Carol. Social motivations for codeswitching: Evidence from Africa. Oxford: Clarendon Press, 1993.

PORTO, Renata Sobrino. Os estudos sociolingüísticos sobre o code-switching: uma revisão bibliográfica. Revista Virtual de Estudos da Linguagem - ReVEL. Vol. 5, n. 9, agosto/2007.

POPLACK, Shana. Sometimes I'll start a sentence in Spanish Y TERMINO EN ESPANOl: toward a typology of code-switching, Linguistics, 18, 1980, p. 581-616.

. Syntactic structure and social function of codeswitching. In: R. P. Durán (Ed.) Latino language and communicative behavior. Norwood, NJ: Ablex, 1981, p. 169-184.

RAMPTON, Ben. Crossing: Language and ethnicity amoung adolescents. Manchester: St. Jerome Press, 2005.

RICHARDSON, Viviane. Portu-English: análise de code-switching português-inglês no discurso colloquial de uma família bilíngue. Dissertação de Mestrado (Estudos da Linguagem). Potifícia Universidade Católica do Rio de Janeiro, 2000.

SAVEDRA, Mônica. Bilinguísmo e bilingualidade: uma nova proposta conceitual. In: SAVEDRA, M.M.G. \& SALGADO, A.C. Sociolingüística no Brasil: uma contribuição dos estudos sobre línguas em/de contato. Rio de Janeiro: 7 Letras, 2009, pp. 121-140.

THOMASON, Sara G. Language contact: an introduction. Edinburgh: Edinburgh University Press, 2001. 
WEINREICH, Uriel. Languages in contact. New York: Linguistic Circle of New York, 1953.

ZINKHAHN RHOBODES, Dagna. The permeability of language borders on the example of German-Polish language mixing. In: ROSENBERG, Peter/JUNGBLUTH, Konstanze, Jungbluth/ ZINKHAHN RHOBODES, Dagna (Eds.) Linguistic construction of ethnic borders. Frankfurt am Main: Peter Lang, 2015.

\section{BRAZILIAN PORTUGUESE AND GERMAN IN CONTACT IN TWO VIRTUAL COMMUNITIES}

ABSTRACT: This paper presents an analysis of codeswitching between Brazilian Portuguese and German language in two virtual communities on Facebook: Brasileiros em Berlim and Brasileiros e Brasileiras em Berlim. We have adopted the concepts of durability, permeability and liminality traced by Zinkhahn-Rhobodes (2015) to observe the permeability of the linguistic border between these two languages.

KEYWORDS: languages in contact; code-switching; linguistic border.

Recebido em: 31/05/2016 Aprovado em: 09/11/2016 